ARTICULO ORIGINAL

\title{
Acción de algunos biocidas sobre las micobacterias no tuberculosas
}

Clara Inés León ${ }^{1}$, Yenny Patricia Pardo ${ }^{2}$, Claudia Ximena Ramírez ${ }^{2}$

${ }^{1}$ Laboratorio de Micobacterias, Subdirección de Investigación y Desarrollo, Instituto Nacional de Salud, Bogotá, D.C., Colombia.

${ }^{2}$ Facultad de Bacteriología, Universidad Colegio Mayor de Cundinamarca, Bogotá, D.C., Colombia

La incidencia de enfermedad y de infecciones nosocomiales por micobacterias no tuberculosas se ha incrementado afectando a pacientes inmunocompetentes y, con mayor frecuencia, a pacientes inmunosuprimidos. Se sabe que las micobacterias son más resistentes a los biocidas que otras bacterias no esporuladas y que algunas especies son particularmente resistentes. Se estudio la acción biocida del hipoclorito de sodio y del glutaraldehído sobre Mycobacterium avium-intracellulare, Mycobacterium gordonae, Mycobacterium fortuitum y Mycobacterium chelonae, para lo cual se utilizó la prueba de Kelsey Maurer modificada: se determinó la concentración inhibitoria mínima (CIM) y el tiempo mínimo de acción de cada uno de ellos sobre las diferentes especies de micobacterias.

La efectividad del hipoclorito de sodio y del glutaraldehído frente a las diferentes especies de micobacterias varió al igual que entre los aislamientos de una misma especie.

La CIM y el tiempo de exposición que fueron efectivos contra todas las micobacterias no tuberculosas estudiadas para que el hipoclorito de sodio destruyerá el 99,99\% de la población fue del $0,2 \%$ durante 5 min y para lograr una efectividad del $100 \%$ del $0,5 \%$ durante 15 min. Para el glutaraldehído, la CIM para destruir el $99,99 \%$ de la población fue del $1 \%$ durante 15 min y para una efectividad del $100 \%$ del $2 \%$ durante 15 min

El hipoclorito de sodio y el glutaraldehído son biocidas efectivos para las micobacterias; el primero tiene un costo reducido y actúa a concentraciones muy bajas, pero por ser corrosivo y oxidante no se puede utilizar en la desinfección de equipos hospitalarios o de laboratorio, lo cual convierte al glutaraldehído en una alternativa segura para la desinfección de este tipo de material, sin perder de vista que puede aparecer resistencia a estos biocidas.

Palabras clave: desinfección, micobacterias, MNT, bioseguridad.

\section{Action of some biocides on non tuberculosis mycobacteria}

The incidence of disease and nosocomial infections produced by non tuberculosis mycobacteria (NTM) has increased in immunocompetent patients, but also and more frequently, in immunosupressed patients. Several studies have disclosed that mycobacteria are more resistant to biocides than non-sporulating bacteria; in addition, some species are particularly resistant. The biocide action of sodium hypochloride and glutaraldehyde on Mycobacterium aviumintracellulare, Mycobacterium gordonae, Mycobacterium fortuitum and Mycobacterium chelonae was studied, using a modified Kelsey Maurer test. For the different species, both the minimal inhibitory concentration (MIC) and the minimal action time were determined. Effectiveness of sodium hypochloride and glutaraldehyde against the different mycobacterial species varied. The same was true for different isolates of the same species. Sodium hypochloride effective MIC and exposure time (killing of $99.99 \%$ of all NTM) were $0.2 \%$ and 5 minutes, respectively. In order to achieve $100 \%$ killing, $0.5 \%$ MIC and 15 minute exposure were needed. In the case of glutaraldehyde, $99.99 \%$ of the bacteria were killed with $1 \%$ MIC and a 15 minute exposure. An effectiveness of $100 \%$ was achieved with a $2 \%$ MIC of glutaraldehyde and a 15 minute exposure. Sodium hypochloride and glutaraldehyde are effective biocides for mycobacteria. The first biocide is cheap and effective at low concentrations, but its corrosive and oxidant nature makes it impossible for use in hospitals or with laboratory equipment. Glutaraldehyde (neither corrosive 
nor oxidant) is a safe alternative for disinfection of this type of equipment. However, it is important to bear in mind that these pathogens may develop resistance to biocides.

Key words: desinfection, mycobacteria, NTM, biosafety.

En Colombia y en el mundo, la incidencia de enfermedad por micobacterias se ha incrementado al igual que las infecciones nosocomiales, tanto por Mycobacterium tuberculosis como por las micobacterias no tuberculosas (MNT), afectando a pacientes inmunocompetentes y, con mayor frecuencia, a pacientes inmunosuprimidos especialmente aquellos con sida (1-4).

Numerosos estudios revelan que las micobacterias son más resistentes a los biocidas que otras bacterias no esporuladas $(5,6)$ y otros trabajos han demostrado que algunas especies mico-bacterianas son particularmente resistentes (7-10). Por tanto, es necesario tomar medidas para mejorar las normas de bioseguridad y evitar la presencia de infecciones nosocomiales producidas por micobacterias, utilizando soluciones biocidas activas contra ellas tanto a nivel hospitalario como en los laboratorios donde se manipulan muestras y cultivos con fines diagnósticos e investigativos (10).

Con ese fin, se hace necesario evaluar en condiciones experimentales la acción desinfectante de sustancias biocidas como paso preliminar para su empleo y, posteriormente, realizar una evaluación en los lugares donde su uso es mayor y rutinario como en hospitales y laboratorios; también, para que las autoridades nacionales hagan una evaluación oficial de los productos y puedan emitir las normas para su utilización (11).

Existen varios métodos para realizar esa evaluación; la mayoría determinan la concentración inhibitoria mínima (CIM) y el tiempo de acción, debido a que los desinfectantes por su naturaleza química no se comportan en forma

Correspondencia:

Clara Inés León, Laboratorio de Micobacterias, Instituto Nacional de Salud, Avenida calle 26 No. 51-60, Bogotá, D.C., Colombia. Tel: 220 0926; cleon@ins.gov.co

Recibido: 15/01/02; aceptado: 06/06/02 homogénea frente a los diferentes microorganismos $(12,13)$. Sin embargo, no existe un método universal para la evaluación de la actividad micobactericida de los desinfectantes $(14,15)$.

Teniendo en cuenta el escaso número de estudios informados en la literatura nacional e internacional sobre este tema, se realizó el presente trabajo para contribuir al establecimiento de normas que permitan mejorar la bioseguridad de las personas que trabajan en el área de la salud, previniendo y ayudando a controlar las infecciones nosocomiales por MNT. El objetivo fue determinar la variabilidad de la acción desinfectante del hipoclorito de sodio y del glutaraldehído frente a diferentes especies de MNT de importancia clínica como son: Mycobacterium avium-intracellulare, Mycobacterium gordonae, Mycobacterium fortuitum y Mycobacterium chelonae, mediante una modificación de la prueba de Kelsey Maurer (16).

\section{Materiales y métodos}

Se realizó un estudio de tipo experimental descriptivo. Se utilizaron tres aislamientos de cada una de las siguientes especies: $M$. aviumintracellulare, (1059, 1057 y 994), M. gordonae (1038, 1026 y 1005), M. fortuitum $(1073,1055$ y 1054) y $M$. chelonae (1079, 1076 y 1071), pertenecientes al cepario de micobacterias del Laboratorio de Micobacterias del Instituto Nacional de Salud de Bogotá, Colombia. Todos los aislamientos procedían de pacientes colombianos y se conservan en medio de Lowenstein-Jensen (LJ) a $4{ }^{\circ} \mathrm{C}$.

Los aislamientos se recuperaron del cepario de micobacterias en el medio de LJ y se confirmó la identificación de especie por métodos fenotípicos estandarizados (17).

Se estudió la acción de dos desinfectantes: el hipoclorito de sodio a concentraciones de 0,2 , $0,5,0,75,1$ y $1,5 \%$ y el glutaraldehído en concentraciones de $0,2,0,5,1$ y $2 \%$. Los tiempos de acción probados para los dos desinfectantes fueron $1,2,5,10$ y $15 \mathrm{~min}$. 


\section{Procedimientos}

Obtención del cultivo líquido de las micobacterias: con el fin de trabajar en iguales condiciones y con cultivos homogéneos de cada uno de los aislamientos, se preparó una suspensión en agua destilada estéril a una concentración cuya opacidad correspondía al tubo 3 de la escala de MacFarland y de ella se sembraron $0,75 \mathrm{ml}$ en $50 \mathrm{ml}$ del medio Sauton Tween albúmina modificado (MSTA); el medio se incubó en agitación a $110 \mathrm{rpm}$ a $37^{\circ} \mathrm{C}$; una vez el cultivo alcanzó el máximo de la fase exponencial, la cual se había determinado previamente en el laboratorio para cada una de las especies estudiadas, se hicieron alícuotas en viales de 1,8 $\mathrm{ml}$ que se almacenaron a $-70^{\circ} \mathrm{C}$.

Valoración de la acción desinfectante: se realizó mediante la prueba de Kelsey Maurer modificada, de la manera previamente descrita $(16,17)$, en la cual se tiene en cuenta la neutralización y remoción del biocida en cada uno de los tiempos estudiados $(10,18)$. La modificación realizada a la técnica original introdujo la utilización de tubos taparrosca de ensayo y no cajas de Petri, convirtiendo la prueba de semicuantitativa a una técnica cuantitativa e introduciendo el porcentaje de supervivencia y de efectividad en el análisis de los resultados y la determinación de la reproducibilidad intraensayo.

De cada uno de los aislamientos conservados a $-70{ }^{\circ} \mathrm{C}$, se preparó una suspensión a una concentración de $1 \mathrm{mg} / \mathrm{ml}$ de masa bacilar en agua destilada estéril, la cual es equivalente a la opacidad del nefelómetro 3 de la escala de MacFarland (suspensión madre). A partir de esta suspensión se realizaron los procedimientos que mencionan a continuación.

Evaluación de la actividad micobactericida de los desinfectantes: se tomó $1 \mathrm{ml}$ de la suspensión madre y se diluyó en $9 \mathrm{ml}$ del desinfectante; se mezcló (dilución final $10^{-1} \mathrm{mg} / \mathrm{ml}$ ) y se dejó actuar el desinfectante durante los diferentes tiempos a temperatura ambiente $\left(25^{\circ} \mathrm{C}\right)$. De esta suspensión, se tomó $1 \mathrm{ml}$ y se diluyó en $9 \mathrm{ml}$ de caldo nutritivo (neutralizante); se mezcló (dilución final $10^{-2} \mathrm{mg} / \mathrm{ml}$ ) y se dejó en reposo durante 30 minutos. Pasado este tiempo, se mezcló nuevamente y se sembraron $0,2 \mathrm{ml}$ en 10 tubos de medio de LJ. Los tubos se incubaron a $37^{\circ} \mathrm{C}$; se realizó el conteo de las unidades formadoras de colonias (UFC) a las dos semanas para $M$. fortuitum y $M$. chelonae y a las cinco para $M$. gordonae y M. avium-intracellulare. En caso de obtener un desarrollo masivo, se informó el crecimiento por cruces: +, alrededor de 100 colonias; ++, crecimiento masivo sin ocupar toda la superficie del medio; +++, crecimiento masivo que ocupa toda la extensión del medio de cultivo (17). Se calculó el promedio de UFC para los 10 tubos.

Determinación de la viabilidad del cultivo de las micobacterias: se determinó simultáneamente con la efectividad del desinfectante. Se tomó 1 $\mathrm{ml}$ de la suspensión madre y se hicieron diluciones progresivas en base 10 en agua destilada estéril hasta una concentración final de $10^{-6} \mathrm{mg} / \mathrm{ml}$; de las diluciones $10^{-5}$ y $10^{-6} \mathrm{mg} / \mathrm{ml}$, se sembraron $0,2 \mathrm{ml}$ en 10 tubos de LJ; se incubaron a $37^{\circ} \mathrm{C}$ y se realizó el conteo de las UFC en la misma forma que en el procedimiento anterior. Se calculó el promedio de UFC para los 10 tubos y con éste se calcularon las UFC presentes en la dilución $10^{-2} \mathrm{mg} / \mathrm{ml}$ que se utilizaron en el cálculo del porcentaje de efectividad del desinfectante.

Determinación de la reproducibilidad intraensayo: se calculó la media aritmética $(\bar{X})$ y la desviación estándar (DE) de cada uno de los ensayos realizados. Cada uno de los aislamientos se evaluó por triplicado. Se aceptó como reproducible el ensayo que mostró un valor de ji cuadrado $\left(\chi^{2}\right)<16,9$ con $\mathrm{p}=0,05$ (19).

Determinación de la efectividad de los desinfectantes: se calculó el porcentaje de supervivencia de las micobacterias ante la acción de los dos desinfectantes; este porcentaje se calculó para cada uno de los tiempos y las concentraciones con el empleo de la siguiente fórmula:

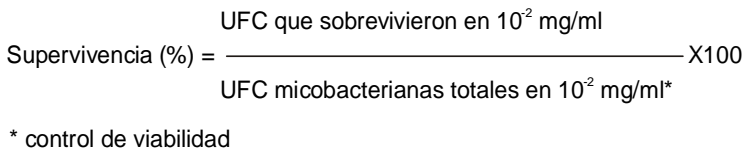

Teniendo en cuenta el porcentaje de supervivencia el cual se consideró que debería ser inferior al $0,01 \%(20,21)$, se calculó el porcentaje de 
efectividad $(\%$ de efectividad $=100-\%$ de supervivencia) para cada desinfectante y para cada una de las especies. Se determinó la CIM que corresponde a la concentración menor del desinfectante capaz de destruir el $99,99 \%$ de la población micobacteriana.

Además, se calculó el tiempo mínimo de acción que corresponde al tiempo menor necesario para destruir el 99,99\%.

\section{Resultados}

Determinación de la efectividad de los desinfectantes: al estudiar el efecto de los desinfectantes sobre cada una de las especies obtuvimos los siguientes resultados.

M. chelonae: la efectividad de la acción desinfectante del hipoclorito de sodio frente a los tres aislamientos de $M$. chelonae no varió, ya que requirió de dos minutos para lograr una mortalidad del 99,99\% a una concentración del $0,2 \%$. La destrucción del $100 \%$ de la población fue igual para todos los aislamientos cuando se utilizó una concentración del 0,2\%, con un tiempo de acción de $10 \mathrm{~min}$.

En cuanto al glutaraldehído, para lograr una mortalidad del $99,99 \%$ de los aislamientos estudiados, se necesitó una concentración del $1 \%$, pero el tiempo de acción varió: para el cultivo 1076 se requirieron $10 \mathrm{~min}$, mientras que para los cultivos 1079 y 1071 tan sólo un minuto. Una mortalidad del $100 \%$ se logró con una concentración del $1 \%$ para los tres cultivos, pero se requirió mayor tiempo de acción (15 min) para el aislamiento 1076.

M. avium-intracelulare: para ser destruidos en un $99,99 \%$, los aislamientos de M. aviumintracelulare necesitaron una concentración de hipoclorito de sodio del 0,2\%. El cultivo 1057 fue más sensible y tan sólo requirió un minuto; los otros cultivos necesitaron dos minutos. Para destruir el $100 \%$ de la población, se necesitó hipoclorito de sodio al 0,2\% durante cinco minutos para todos los aislamientos.

Una concentración de $0,5 \%$ de glutaraldehído destruyó el 99,99\% de los aislamientos en 15 minutos; el cultivo 1059 tan sólo requirió 10 minutos. Una concentración del $1 \%$ de glutaraldehído durante 15 minutos fue suficiente para lograr una mortalidad del $100 \%$, excepto para el cultivo 1057 que requirió 10 min, lo que demostró una menor resistencia de este cultivo al glutaraldehído.

M. fortuitum: la concentración necesaria de hipoclorito de sodio para lograr una mortalidad del $99,99 \%$ fue de $0,2 \%$, pero el tiempo varió: para uno de los aislamientos fue de dos minutos y de cinco para los otros dos. La eliminación del $100 \%$ de la población se logró con una concentración del $0,2 \%$ para los tres cultivos, pero el tiempo de acción varió: para los cultivos 1073 y 1055 fue de 15 minutos y para el 1054 de cinco minutos, lo cual evidenció una resistencia menor de este aislamiento al hipoclorito de sodio.

En cuanto al efecto biocida del glutaraldehído, la CIM para destruir el $99,99 \%$ fue de $0,5 \%$ con tiempos variables para cada aislamiento: entre dos y 10 minutos. La efectividad del glutaraldehído para destruir el $100 \%$ de la población se logró con una concentración del $0,5 \%$ para los aislamientos 1073 y 1054 , mientras que para la 1055 se necesitó una concentración del $1 \%$ durante 15 minutos.

M. gordonae: para eliminar el $99,99 \%$ de la población de los tres cultivos estudiados de $M$. gordonae, se necesitó una concentración de hipoclorito de sodio del $0,2 \%$ durante cinco minutos, mientras que para lograr un efecto micobactericida del $100 \%$, una concentración del $0,5 \%$ durante 10 minutos fue suficiente excepto para el cultivo 1038 que necesitó 15 minutos.

Una concentración del 1\% de glutaraldehído con tiempos variables de exposición (10 y $15 \mathrm{~min}$ ) fue capaz de destruir el 99,99\% de la población de los cultivos 1005 y 1026 . El aislamiento 1038 se mostró más sensible, pues se necesitó una concentración de $0,5 \%$ durante dos minutos.

Al evaluar la mortalidad del $100 \%$, el efecto micobactericida del glutaraldehído sobre $M$. gordonae fue muy variable en los tres aislamientos estudiados, necesitándose una concentración del $2 \%$ durante 15 minutos para poder asegurar que se lograra este efecto sobre cualquiera de ellos.

En los cuadros 1 y 2 se encuentran las CIM y los tiempos de acción del hipoclorito de sodio y del 
glutaraldehído con los que se obtuvieron mortalidades del $99,99 \%$ y del $100 \%$ de la población micobacteriana para cada una de las especies de MNT estudiadas. El tiempo de acción corresponde al requerido para lograr la mortalidad de los tres aislamientos de cada una de las especies estudiadas.

Teniendo en cuenta que se debe elegir para la desinfección una concentración y un tiempo de exposición que sean efectivos contra todas las MNT estudiadas, se determinó que la CIM para que el hipoclorito de sodio destruya el $99,99 \%$ de la población fue del $0,2 \%$ durante cinco minutos y para lograr una efectividad del $100 \%$ una concentración de $0,5 \%$ durante 15 minutos (cuadro 1). Para el glutaraldehído, la CIM para destruir el $99,99 \%$ de la población fue del $1 \%$ durante 15 minutos y para una efectividad del $100 \%$ se necesitó una concentración del $2 \%$ durante 15 minutos (cuadro 2).
Para que el hipoclorito de sodio alcance una efectividad del $100 \%$ sobre las MNT en menor tiempo se debe utilizar al $0,75 \%$ durante un minuto (cuadro 1) y para el glutaraldehído una concentración del $2 \%$ durante 15 minutos (cuadro 2).

Reproducibilidad intraensayo: Ia constante similitud de los resultados obtenidos en cada uno de los ensayos realizados, cada vez que se valoró tanto el hipoclorito de sodio como el glutaraldehído a las diferentes concentraciones y tiempos, mostraron una alta reproducibilidad intraensayo sustentada por los siguientes indicadores: todos los recuentos de UFC de cada experimento se encontraron ubicados en el rango de +2 DE y el valor de $X^{2}$ en cada ensayo fue $\leq 16,9$.

\section{Discusión}

La evaluación de los diferentes micobactericidas no ha sido consistente a través de los diferentes

Cuadro 1. Concentraciones y tiempos de acción del hipoclorito de sodio sobre las micobacterias no tuberculosas.

\begin{tabular}{lcccc}
\hline Especie & \multicolumn{2}{c}{$\begin{array}{c}\text { Efectividad } \\
\mathbf{9 9 , 9 9 \%}\end{array}$} & $\begin{array}{c}\text { Efectividad } \\
\mathbf{1 0 0 \%}\end{array}$ \\
& $\begin{array}{c}\text { Concentración } \\
\%\end{array}$ & $\begin{array}{c}\text { Tiempo } \\
\text { min }\end{array}$ & $\begin{array}{c}\text { Concentración } \\
\%\end{array}$ & $\begin{array}{c}\text { Tiempo } \\
\text { min }\end{array}$ \\
\hline M. chelonae & 0,2 & 2 & 0,2 & 10 \\
M. avium-intracellulare & 0,2 & 2 & 0,2 & 5 \\
M. fortuitum & 0,2 & 5 & 0,2 & 15 \\
M. gordonae & 0,2 & 5 & 0,5 & 15 \\
MNT* & 0,2 & 5 & 0,5 & 15 \\
Recomendación & 0,2 & 5 & 0,75 & 1 \\
\hline
\end{tabular}

${ }^{*}$ MNT: micobacterias no tuberculosas

Cuadro 2. Concentraciones y tiempos de acción del glutaraldehído sobre las micobacterias no tuberculosas.

\begin{tabular}{|c|c|c|c|c|}
\hline \multirow[t]{2}{*}{ Especie } & \multicolumn{2}{|c|}{$\begin{array}{c}\text { Efectividad } \\
99,99 \%\end{array}$} & \multicolumn{2}{|c|}{$\begin{array}{c}\text { Efectividad } \\
100 \%\end{array}$} \\
\hline & $\begin{array}{c}\text { Concentración } \\
\%\end{array}$ & $\begin{array}{l}\text { Tiempo } \\
\text { min }\end{array}$ & $\begin{array}{c}\text { Concentración } \\
\%\end{array}$ & $\begin{array}{c}\text { Tiempo } \\
\text { min }\end{array}$ \\
\hline M. chelonae & 1,0 & 10 & 1,0 & 15 \\
\hline M. avium-intracellulare & 0,5 & 15 & 1,0 & 15 \\
\hline M. fortuitum & 0,5 & 10 & 1,0 & 15 \\
\hline M. gordonae & 1,0 & 15 & 2,0 & 15 \\
\hline MNT§ & 1,0 & 15 & 2,0 & 15 \\
\hline Recomendación & 2,0 & 5 & 2,0 & 15 \\
\hline
\end{tabular}

$\S$ MNT: micobacterias no tuberculosas 
estudios realizados debido a problemas metodológicos y a la resistencia intrínseca de la pared de la micobacteria (10). Teniendo en cuenta los resultados anteriores y que aún no existe una técnica estandarizada confiable, la técnica de Kelsey Maurer modificada utilizada en este trabajo podría ser una buena opción para que se adopte en la evaluación de los micobactericidas.

La metodología empleada brinda confiabilidad con respecto a la información obtenida de la acción biocida del hipoclorito de sodio y del glutaraldehído frente a las MNT estudiadas y cumple con las recomendaciones dadas por la Association of Official Analytical Chemists (AOAC) para la evaluación de desinfectantes $(21,22)$. Además, por ser una técnica cuantitativa permite comparaciones con otros desinfectantes o con su acción sobre otras micobacterias. El tiempo de incubación utilizado tanto para MNT de crecimiento rápido como lento fue el adecuado y está de acuerdo con las recomendaciones que se deben tener en cuenta al respecto, ya que uno de los problemas en la diferencia de los resultados de los distintos desinfectantes que actúan como micobactericidas, es la utilización de tiempos de incubación demasiado cortos que no permiten la visualización del crecimiento, lo cual da resultados erróneos $(13,18)$; además, se utilizó un neutralizante que detiene la acción del desinfectante para poder establecer con certeza el tiempo de acción.

Se obtuvo una muy buena reproducibilidad intraensayo e interensayo, atribuible en parte al empleo de cultivos líquidos homogéneos de condiciones iguales de los diferentes aislamientos, lo cual corrobora lo demostrado en este aspecto por Bloomfield y colaboradores (15).

Nuestro estudio muestra que existen diferencias en la sensibilidad de las distintas especies micobacterianas a los biocidas estudiados; las más resistentes fueron $M$. fortuitum y $M$. gordonae para el hipoclorito de sodio y para el glutaraldehído, $M$. chelonae y $M$. gordonae, lo cual está de acuerdo con otro estudio en el que se vio que la sensibilidad de Mycobacterium bovis var. BCG, de Mycobacterium terrae y de $M$. chelonae al fenol y a desinfectantes de tipo aldehído varía de una especie a otra (23).
Estas diferencias han llevado a varios autores a sugerir la utilización de $M$. avium-intracelulare o de $M$. chelonae como organismos indicadores para determinar la concentración y el tiempo de acción de los posibles micobactericidas y no utilizar $M$. tuberculosis o M. smegmatis que son más sensibles y que se están utilizando como organismos indicadores (24-26).

En un estudio anterior realizado en nuestro laboratorio, se determinó la acción de los mismos desinfectantes sobre $M$. tuberculosis y se encontró que con el hipoclorito de sodio se requiere una concentración mayor, pero un tiempo de acción más corto que para las MNT. Para el glutaraldehído, la concentración fue del $2 \%$ pero durante un período de 10 minutos (16), lo cual nos demostró que, en realidad, $M$. tuberculosis es más sensible a este biocida que algunas de las MNT, lo que confirma que $M$. tuberculosis no es el organismo indicador apropiado para la evaluación de los micobactericidas (13). Podría pensarse en la posibilidad de trabajar con dos micobacterias indicadoras: M.tuberculosis y una MNT. Otra posibilidad sería utilizar Mycobacterium terrae en lugar de M. tuberculosis, que es una micobacteria de crecimiento intermedio con similar resistencia a ciertos desinfectantes, como aldehídos, fenoles y ácido peracético, que M. tuberculosis (23); sin embargo, es recomendable utilizar otra MNT de mayor resistencia como M. gordonae.

Las diferencias encontradas en la acción antimicrobiana de los biocidas estudiados sobre los aislamientos micobacterianos de una misma especie, se debieron posiblemente a la aparición de mutantes espontáneas, lo cual implica un cambio en sus características genéticas que trae consigo variaciones fenotípicas que le permiten sobrevivir en presencia de un compuesto químico haciéndolas resistentes a éste (13). La resistencia intrínseca o innata generalmente es de dos tipos: por inactivación enzimática o por disminución de la permeabilidad celular; en un estudio realizado por Manzoor y colaboradores (9), los investigadores asumieron que los cambios en la estructura o en la composición de la pared celular que la hacen hidrofóbica se pueden deber a un incremento en la producción de ácidos micólicos 
o de ácidos grasos de cadena corta, pero al comparar cepas resistentes y sensibles al glutaraldehído no encontraron diferencias, lo cual demostró que no juegan un papel importante en la resistencia a esta sustancia. Sin embargo, demostraron cómo una disminución en los monosacáridos del arabinogalactán de la pared se asoció con un incremento en la resistencia al glutaraldehído (9).

La resistencia también puede ser adquirida ya sea por mutación, adquisición de plásmidos o transposones; por tanto, resulta necesario realizar investigaciones a nivel molecular y metabólico para determinar con certeza la causa de esta posible resistencia (13).

Los resultados de la variabilidad de la acción desinfectante del glutaraldehído para los diferentes aislamientos de $M$. chelonae encontrada por nosotros está de acuerdo con otros informes acerca de la resistencia que pueden presentar algunos aislamientos de $M$. chelonae al glutaraldehído $(9,23)$. Esta fue una de las razones para el desarrollo del ortoparaldehído como biocida, con muy buenos resultados hasta el momento y con eficacia como micobactericida (23).

Los desinfectantes en la práctica se deben utilizar a concentraciones previamente determinadas en el laboratorio de acuerdo con los microorganismos que se requiera eliminar, pero se debe tener en cuenta que la desinfección debe ser efectiva y rápida, por lo cual se debe emplear una mayor concentración de desinfectante que la CIM y un tiempo menor de acción. En este estudio, para que el hipoclorito de sodio alcanzara una efectividad del $100 \%$ sobre las MNT en menor tiempo, se debió utilizar al $0,75 \%$ durante un minuto (cuadro 1). Para el glutaraldehído, se debe usar una concentración del $2 \%$ durante 15 minutos y determinar su acción a concentraciones mayores y menor tiempo, ya que en el estudio fue la concentración más alta (cuadro 2).

Sin embargo, es importante no olvidar que la efectividad de los desinfectantes se puede ver afectada por cambios en el $\mathrm{pH}$, la temperatura y la presencia de materia orgánica $(10,20)$, factores que deben ser evaluados. En este estudio, el hipoclorito de sodio se presenta como un buen desinfectante por ser efectivo a concentraciones bajas y con un tiempo de acción muy corto, pero la presencia de materia orgánica puede causar una ligera reducción de su efectividad lo mismo que un mal almacenamiento, que permita que el cloro escape, por lo cual es importante reemplazar frecuentemente el hipoclorito de los recipientes donde se descarta material y así asegurar una desinfección adecuada (14).

En conclusión, el hipoclorito de sodio y el glutaraldehído son sustancias antimicobacterianas efectivas tanto para MNT como para $M$. tuberculosis; el primero tiene un costo reducido y actúa a concentraciones muy bajas, pero por ser un agente corrosivo y oxidante no se puede utilizar en la desinfección de equipos de terapia respiratoria, broncoscopios, endoscopios, instrumental quirúrgico y odontológico, convirtiéndose el glutaraldehído en una alternativa segura para la desinfección de este tipo de material siendo, además, efectivo para destruir otros microorganismos vegetativos como Pseudomonas aeruginosa, Serratia marscenses, hongos y virus como Herpes simplex tipo 1 y 2 , Adenovirus tipo 2 y otros $(27,28)$. Es de vital importancia realizar una vigilancia continua de la acción de los diferentes desinfectantes sobre las micobacterias que están circulando, para poder utilizar concentraciones más altas o nuevos desinfectantes que puedan ser efectivos y se disponga de una alternativa para la desinfección, especialmente de equipos quirúrgicos y para evitar así las infecciones nosocomiales por estas micobacterias.

\section{Agradecimientos}

A Elizabeth Castañeda del Laboratorio de Microbiología de la Subdirección de Investigación y Desarrollo del Instituto Nacional de Salud por sus aportes en la revisión y sugerencias al documento final.

\section{Referencias}

1. León $\mathbf{C l}$. Micobacterias no tuberculosas asociadas al VIH en Colombia. Biomédica 1997;17:42-4.

2. León $\mathrm{Cl}$, Guerrero MI, De la Hoz F, Murcia MI, Martínez R, Jiménez M, et al. Tuberculosis VIH/AIDS relation in Colombia. Spanish J Chemother 1997;10:153. 
3. Crespo MP, Corral R, Alzate A. El diagnóstico de la infección por micobacterias en individuos $\mathrm{VIH}(+)$. Colombia Médica 1994;25:86-91.

4. Sterling TR, Moore RD, Graham NM, Astemborski J, Vlahov D, Chisson RE. Mycobacterium tuberculosis infection and disease are not associated with protection against subsequent disseminated $M$. avium complex disease. AIDS 1998;12:1451-7.

5. Carson LA, Petersen NJ, Favero MS, Agüero SM. Growth characteristics of atypical mycobacteria in water and their comparative resistance to desinfectants. Appl Environ Microbiol 1978;38:839-46.

6. Jarlier V, Nikaido H. Mycobacterial cell wall: structure and role in natural resistance to antibiotics. FEMS Microbiol Letter 1994;123:11-8.

7. Croshaw B. The destruction of mycobacteria. En: Hugo WB, editor. Inhibition and destruction of the microbial cell. London: Academic Press; 1971. p.420-49.

8. Griffths PA, Babb JR, Bradley CR, Fraise AP. Glutaraldehide-resistant $M$. chelonae from endoscope washer desinfectors. J Appl Microbiol 1997;82:519-26.

9. Manzoor SE, Lambert PA, Griffiths PA, Gill MJ, Fraise AP. Reduced glutaraldehyde susceptibility in Mycobacterium chelonae associated with altered cell wall polysaccharides. J Antimicrob Chemoter 1999;43:759-65.

10. Rikimaru T, Kondo M, Kondo S, Oizumi K. Efficacy of common antiseptics against mycobacteria. Int J Tuber Lug Dis 2000;4:570-6.

11. Scopetti F, Casal M, Chiaradonna P, Darbord C, Katila ML, Hoffner S, et al. An European colaborative study to produce a common protocol for the evaluation of micobactericidal activity of desinfectants. Spanish $J$ Chemother 1997;10:30.

12. Astudillo M, Concha A, Daza LE. Desinfectantes hospitalarios: cómo evaluarlos y propuesta para estandarizar su valoración. Colombia Médica 1992;23:162-7.

13. Russell AD. Activity of biocides against mycobacteria. J Appl Bacteriol 1996;81(Suppl):87s-101s.

14. Best M, Sattar SA, Springthorpe VS, Kennedy ME. Efficacies of selected desinfectants against Mycobacterium tuberculosis. J Clin Microbiol 1990;28:2234-9.

15. Bloomfield SF, Looney E. Evaluation of the repeatability and reproducibility of European suspension test methods for antinmicrobial activity of desinfectants and antiseptics. J Appl Microbiol 1992;73:87-93.
16. León $\mathbf{C l}$, Ulloa de Moreno Al, Orozco LC. Efecto de algunos desinfectantes sobre el $M$. tuberculosis. Serie Memorias de Eventos Científicos Colombianos. Bogotá: ICFES; 1986. p.84.

17. Kent T, Kubica GP. Identification test techniques. En: U.S. Departament of Health and Human Services. Public health Mycobacteriology. A guide for the level III laboratory. Atlanta: Centers for Disease Control; 1985. p.71-120.

18. Cole EC, Rutala WA, Nessen L, Wannamaker NS, Weber DJ. Effect of methodology, dilution and exposure time on the tuberculocidal activity of glutaraldehydebased desinfectants. Appl Environ Microbiol 1990;56:1813-7.

19. OMS. In vitro assays of BCG products. OMS Technical Guide 1980; 77.9: 2-45.

20. Maurer I. Hospital hygiene. Great Britain: Edward Arnold Publishers Ltd.; 1974.

21. Rutala WA. APIC guideline for selection and use of disinfectants. American J Infect Control 1996;24:31342.

22. Environmental Protection Agency. Tuberculocidal activity of disinfectants. AOAC Official Method 96512. Cincinnatti: Environmental Protection Agency; 1988.

23. Gregory AW, Schaalje B, Smart JD, Robison RA. The mycobactericidal efficacy of ortho-phthalaldehyde and the comparative resistances of Mycobacterium bovis, Mycobacterium terrae, and Mycobacterium. chelonae. Infect Control Hosp Epidemiol 1999;20:324-30.

24. Collins FM. Bactericidal activity of alkaline glutaraldehyde solution against a number of atypical mycobacterial species. J Applied Bacteriol 1986;61:24751.

25. van Klingeren B, Pullen W. Glutaraldehyde resistant mycobacteria from endoscope washers. J Hosp Infect 1993;25:147-9.

26. Nye K, Chadna DK, Hodgkin P, Bradley C, Hancox J, Wise R. Mycobacterium chelonae isolation from bronchoalveolar lavage fluid and its practical implications. J Hosp Infect 1990;16:257-61.

27. Jette LP, Ringuette L, Ishak M, Miller M, Saint Antoine P. Evaluation of three glutaraldehyde based desinfectants used in endoscopy. J Hosp Infect 1991;30:295-303.

28. Rodríguez Frojan G, Castella J, Puzo C. Desinfección del broncoscopio con glutaraldehído fenolato a la dilución 1:8. Arch Broconeumol 1994;30:485-8. 